\title{
ANALYTIC SOLUTION OF THE LINEARIZED SHALLOW-WATER WAVE EQUATIONS FOR CERTAIN CONTINUOUS DEPTH VARIATIONS
}

\author{
D. L. CLEMENTS and C. ROGERS
}

(Received 17 July, 1974)

(Revised 26 September, 1974)

\begin{abstract}
The linear long-wave equations with (and without) small ground motion are considered. The governing equations are represented in a matrix form and transformations are sought which reduce the system to (for example) a form associated with the conventional wave equation. Integration of the system is then immediate. It is shown that such a reduction may be achieved provided the variation in water depth is specified by certain multi-parameter forms.
\end{abstract}

\section{Introduction}

The majority of analytical work on two-dimensional wave generation in shallow water has been restricted to either constant depth situations (e.g. Kajiura [1]) or cases when the bottom has uniform slope (e.g. Tuck and Hwang [2]).

The purpose of the present investigation is to obtain analytical solutions to the linearized long-wave equation for cases in which the bottom surface is curved. The technique employed is to transform the relevant governing equation to one associated with either the constant depth or uniform slope situations. All the analytical results for these cases can thus be employed and then the inverse transformations yield information regarding wave propagation in shallow water with a curved bottom profile. This procedure does not, of couse, work for an arbitrary bottom profile, but is dependent upon the bottom being specified by certain multi-parameter forms which emerge in the course of the analysis. The parameters in these forms may be chosen to fit locally the analytical curve to actual curvilinear inclines.

It is noted that although the approach presented here is believed new in the theory of water waves, a somewhat similar approach has been utilised by the authors in other fields, for instance, in the theory of elastic and elastic-plastic wave propagation (Clements and Rogers [3,4], Rogers and Clements [5]). 


\section{The shallow-water equations with ground motion}

The equations of shallow-water or long-wave theory for arbitrary ground motion are available in Tuck and Hwang [2] and adopt the form

$$
\begin{gathered}
\frac{\partial}{\partial t}(\eta+h)+\frac{\partial}{\partial x}[u(\eta+h)]=0, \\
\frac{\partial u}{\partial t}+u \frac{\partial u}{\partial x}=-g \frac{\partial \eta}{\partial x},
\end{gathered}
$$

where $u(x, t)$ is the horizontal velocity component. The equations of the bottom and surface are respectively given by

$$
\begin{aligned}
& y=-h(x, t), \\
& y=\eta(x, t),
\end{aligned}
$$

in the coordinate system of Figure 1. If it is taken that an upward bottom displacement of magnitude $\eta_{0}(x, t)$ occurs where the undisturbed bottom shape is $y=-h_{0}(x)$, we may write

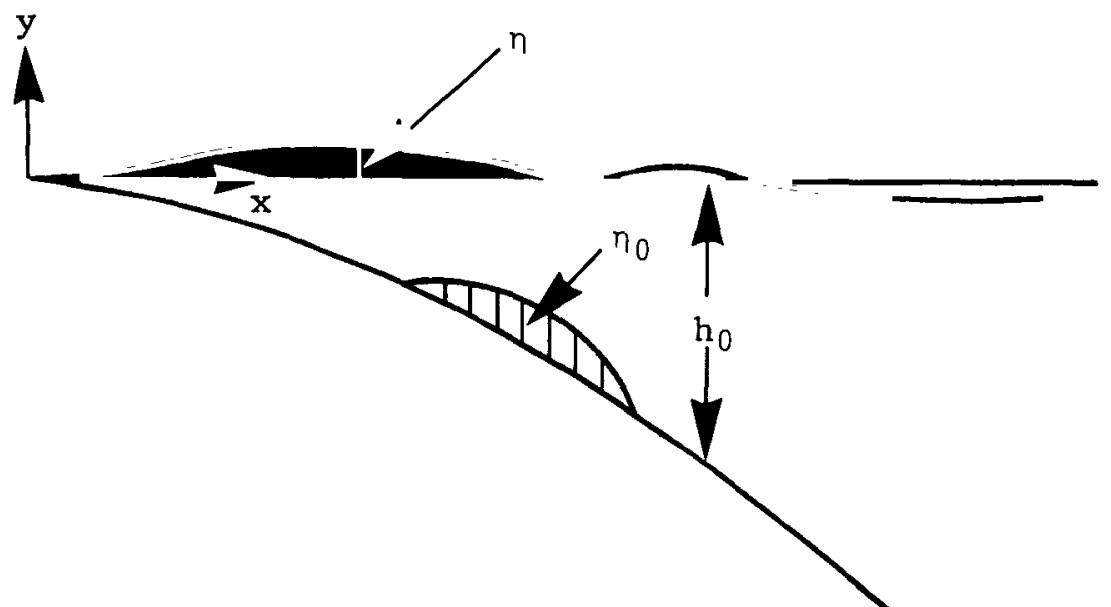

Figure 1.- Schematic drawing of the ground motion and symbol definitions.

$$
h(x, t)=h_{0}(x, t)-\eta_{0}(x, t),
$$

so that (2.1) becomes

$$
\frac{\partial \eta}{\partial t}+\frac{\partial}{\partial x}\left[u\left(h_{0}+\eta\right)\right]=\frac{\partial \eta_{0}}{\partial t}+\frac{\partial}{\partial x}\left(u \eta_{0}\right) .
$$

If attention is restricted to small amplitude waves (so that $\eta$ and $\eta_{0}$ are small compared with $h_{0}$ and $u$ is small compared with the local wave speed $\left.\left(g h_{0}\right)^{\frac{1}{2}}\right)$ 
equations (2.2) and (2.6) may be linearized to obtain the shallow water equations

$$
\begin{gathered}
\frac{\partial u}{\partial t}+g \frac{\partial \eta}{\partial x}=0 \\
\frac{\partial \eta}{\partial t}+\frac{\partial}{\partial x}\left(u h_{0}\right)=\frac{\partial \eta_{0}}{\partial t}
\end{gathered}
$$

incorporating the forcing term $\partial \eta_{0} / \partial t$ due to ground motion.

If we now set

$$
\begin{aligned}
& u^{*}=-u h_{0}(x), \\
& \eta^{*}=\eta,
\end{aligned}
$$

the system (2.7), (2.8) can be written in the convenient matrix form

$$
\mathbf{\Omega}_{x}^{*}=\boldsymbol{\Lambda} \boldsymbol{\Omega}_{t}^{*}+\boldsymbol{E}_{t}^{*}
$$

where subscripts denote partial derivatives and

$$
\begin{aligned}
\mathbf{\Omega}^{*} & =\left(\begin{array}{c}
\eta^{*} \\
u^{*}
\end{array}\right), \\
\boldsymbol{\Lambda} & =\left(\begin{array}{cc}
0 & 1 /\left(g h_{0}\right) \\
1 & 0
\end{array}\right), \\
\boldsymbol{E}^{*} & =\left(\begin{array}{c}
0 \\
-\eta_{0}
\end{array}\right) .
\end{aligned}
$$

Introduction of the new independent variables $x^{*}, t *$ according to

$$
\begin{aligned}
x^{*} & =\int\left(g h_{0}\right)^{-\frac{1}{2}} d x, \\
t^{*} & =t,
\end{aligned}
$$

transforms $(2.11)$ to

$$
\boldsymbol{\Omega}_{x^{*}}^{*}=\boldsymbol{H}^{*} \boldsymbol{\Omega}_{1 *}^{*}+\boldsymbol{E}_{\imath^{*}}^{*},
$$

where

$$
\begin{gathered}
\boldsymbol{H}^{*}=\left(\begin{array}{cc}
0 & c^{-1} \\
c & 0
\end{array}\right), \\
\boldsymbol{E}^{*}=\left(\begin{array}{c}
0 \\
-\eta_{0}^{*}
\end{array}\right),
\end{gathered}
$$

with

$$
\begin{gathered}
c^{2}=g h_{0}(x), \\
\eta_{0}^{*}=c \eta_{0} .
\end{gathered}
$$




\section{The matrix transformations}

In this section, certain matrix transformations are constructed which transform the system (2.17) to one of two "canonical forms", namely

$$
\mathbf{\Omega}_{x^{\prime}}^{\prime}=\boldsymbol{H}^{\prime} \boldsymbol{\Omega}_{t^{\prime}}^{\prime}+\boldsymbol{E}_{t^{\prime}}^{\prime},
$$

where, either

$$
\boldsymbol{H}^{\prime}=\left(\begin{array}{ll}
0 & 1 \\
1 & 0
\end{array}\right)
$$

or

(ii)

$$
\boldsymbol{H}^{\prime}=\left(\begin{array}{cc}
0 & \left(g \alpha x^{\prime}\right)^{-\frac{1}{2}} \\
\left(g \alpha x^{\prime}\right)^{\frac{1}{2}} & 0
\end{array}\right)
$$

The case (i) is essentially that corresponding to the constant depth situation, while (ii) is the uniform slope case investigated by Truck and Hwang [2]. In particular, in the absence of ground motion, for case (i), (3.1) reduces to a form associated with the conventional wave equation. It emerges that such transformations may be constructed subject to $h_{0}(x)$ adopting a variety of forms which involve parameters available for curve fitting.

We consider the transformations

$$
\begin{gathered}
\boldsymbol{\Omega}_{x^{\prime}}^{\prime} \boldsymbol{A} \boldsymbol{\Omega}_{x^{*}}^{*}+\boldsymbol{B} \mathbf{\Omega}^{*}, \quad|\boldsymbol{A}| \neq 0, \\
\mathbf{\Omega}_{t^{\prime}}^{\prime}=\tilde{\boldsymbol{A}} \boldsymbol{\Omega}_{t^{*}}^{*}+\tilde{\boldsymbol{B}} \boldsymbol{\Omega}^{*}, \quad|\tilde{\boldsymbol{A}}| \neq 0, \\
\boldsymbol{x}^{\prime}=x^{*}, \\
\boldsymbol{t}^{\prime}=t^{*},
\end{gathered}
$$

where $\boldsymbol{A}, \boldsymbol{B}, \tilde{\boldsymbol{A}}, \tilde{\boldsymbol{B}}$ are $2 \times 2$ matrices with entries functions of $x *$ designated by $\left[a_{i}^{i}\right],\left[b_{j}^{i}\right],\left[\tilde{a}_{j}^{i}\right]$ and $\left[\tilde{b}_{j}^{i}\right]$ respectively. Transformations of the type (3.4)-(3.5) are sought which transform

$$
\boldsymbol{\Omega}_{x^{\prime}}^{\prime}=\boldsymbol{H}^{\prime} \boldsymbol{\Omega}_{t^{\prime}}^{\prime}+E_{t^{\prime}}^{\prime} \leftrightarrow \boldsymbol{\Omega}_{x^{*}}^{*}=H^{*} \boldsymbol{\Omega}_{t^{*}}^{*}+E_{t^{*}}^{*}
$$

where $H^{*}$ is defined by (2.18) and $H^{\prime}$ adopts the form (3.2) or (3.3).

It is assumed that $\eta^{*}, u^{*}, \eta^{\prime}, u^{\prime}$ have continuous mixed second order derivatives with respect to the independent variables $x^{*}, t^{*}$ so that the commutativity conditions

$$
\begin{aligned}
\boldsymbol{\Omega}_{x^{*} t^{*}}^{*} & =\boldsymbol{\Omega}_{t^{-x^{*}}}^{*}, \\
\boldsymbol{\Omega}_{x^{\prime} t^{\prime}}^{\prime} & =\boldsymbol{\Omega}_{t^{\prime} x^{*}}^{\prime},
\end{aligned}
$$

obtain. The conditions (3.9) and (3.10) may be used in conjunction with the relations (3.4)-(3.7) to yield 


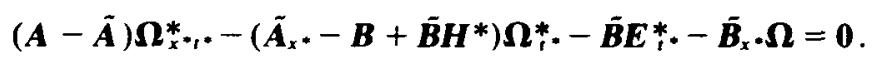

This equation will be satisfied if

$$
\begin{gathered}
\boldsymbol{A}=\tilde{\boldsymbol{A}}, \\
\tilde{\boldsymbol{B}}_{x^{*}}=\mathbf{0}, \\
\tilde{\boldsymbol{B}} \boldsymbol{E}_{\imath *}^{*}=\mathbf{0}, \\
\tilde{\boldsymbol{A}}_{x^{*}}-\boldsymbol{B}+\tilde{\boldsymbol{B}} \boldsymbol{H}^{*}=\mathbf{0},
\end{gathered}
$$

while the transformation (3.8) is effected if

$$
\begin{aligned}
\boldsymbol{B} & =\boldsymbol{H}^{\prime} \tilde{\boldsymbol{B}}, \\
\boldsymbol{E}_{t^{\prime}}^{\prime} & =\boldsymbol{A} \boldsymbol{E}_{{ }^{*}}^{*}, \\
\boldsymbol{H}^{*} & =\tilde{\boldsymbol{A}}^{-1} \boldsymbol{H}^{\prime} \tilde{\boldsymbol{A}} .
\end{aligned}
$$

Thus the transformation from (2.17) to (3.1) is achieved via the relations $(3,4)-(3.7)$ provided the conditions $(3.11)-(3.17)$ hold.

It is now convenient to choose the matrix $A$ in the form

$$
\boldsymbol{A}=\left(\begin{array}{cc}
a_{1} & 0 \\
0 & a_{2}^{2}
\end{array}\right) \text {, }
$$

so that the matrix $\tilde{\boldsymbol{B}}$ is given by

$$
\tilde{\boldsymbol{B}}=\left(\begin{array}{cc}
0 & \tilde{b}_{2}^{1} \\
\tilde{b}_{1}^{2} & 0
\end{array}\right)
$$

where $\tilde{b}_{2}^{1}$ and $\tilde{b}_{1}^{2}$ are constants. Hence, using (3.17) it is apparent that $\boldsymbol{H}^{\prime}$ adopts the form

$$
\boldsymbol{H}^{\prime}=\boldsymbol{A H}^{*} \boldsymbol{A}^{-1}=\left(\begin{array}{cc}
0 & a_{1}^{1} h_{2}^{\prime *} / a_{2}^{2} \\
a_{2}^{2} h_{1}^{2 *} / a_{1}^{1} & 0
\end{array}\right)
$$

while (3.14) yields

$$
\begin{aligned}
& \left(a_{1}^{1}\right)_{x}=-h_{2}^{1} \tilde{b}_{1}^{2}+h_{1}^{2} \tilde{b}_{2}^{1}\left(a_{1}^{1} / a_{2}^{2}\right)=0, \\
& \left(a_{2}^{2}\right)_{x^{*}}-h_{1}^{2} \tilde{b}_{2}^{1}+h_{2}^{1} \tilde{b}_{1}^{2}\left(a_{2}^{2} / a_{1}^{1}\right)=0 .
\end{aligned}
$$

Combination of these equations yields

$$
\operatorname{det} \boldsymbol{A}=a a_{2}^{2}=\lambda \text { (constant), } \lambda \neq 0,
$$

so that the system (3.21), (3.22) may be reduced to the single equation in $a_{1}$

$$
\left(a_{1}^{1}\right)_{x^{*}}+h_{1}^{2 \prime} \tilde{b}_{2}^{1} \lambda^{-1}\left(a_{1}^{1}\right)^{2}-h_{2}^{1 \prime} \tilde{b}_{1}^{2}=0 \text {. }
$$

The cases where there is, and is not, ground motion present are discussed in turn in (a) and (b) below. 
(a) Ground motion PRESENT

In this case, from (2.19) and (3.13), $\bar{b}_{2}^{1}=0$ so that (3.24) reduces to

$$
\left(a_{1}^{\prime}\right)_{x^{*}}-h_{2}^{\prime \prime} b_{1}^{2}=0 \text {. }
$$

In the case when $H^{\prime}$ is given by $(3,2), h_{2}^{\prime \prime}=1$ so that $(3.25)$ yields

$$
a_{!}^{1}=\tilde{b}_{!}^{2} x^{*}+\delta
$$

where $\delta$ is a constant. Now from (2.18), (2.20), (3.17) and (3.26) it follows that

$$
g h_{0}=\left(a_{1}^{1}\right)^{4} / \lambda^{2}=\left(b_{1}^{2} x^{*}+\delta\right)^{4}
$$

and hence, using (2.15)

$$
h_{0}=(A x+B)^{\frac{4}{3}}
$$

where $A$ and $B$ are constants.

When $H^{\prime}$ is given by (3.3), $h_{2}^{\prime \prime}=\left(g \alpha x^{\prime}\right)^{-\frac{1}{2}}$ so that (3.25) yields

$$
a_{1}^{\prime}=2 \tilde{b}_{1}^{2}(g \alpha)^{-\frac{1}{2}} x * \frac{1}{2}+\delta .
$$

Hence, from (2.18), (2.20), (3.17) and (3.28) we obtain

$$
\begin{aligned}
h_{0}=\left(g \lambda^{2}\right)^{-1}\left(g \alpha x^{*}\right)(a !)^{4} \\
=\alpha x *\left[2 \tilde{b}_{1}^{2}(g \alpha)^{-\frac{1}{2}} x^{* \frac{1}{2}}+\delta\right]^{4} / \lambda^{2} .
\end{aligned}
$$

For this case the form of $h_{0}(x)$ is, in general, available only in the parametric form described by (3.29) together with the $\left(x^{*}, x\right)$-relation

$$
2\left[12\left(\tilde{b}_{1}^{2}\right)^{2} x *+15(g \alpha)^{\frac{1}{2}} \tilde{b}_{1}^{2} \delta x^{* \frac{1}{2}}+5(g \alpha) \delta^{2}\right] x^{* \frac{3}{2}}=15(g \alpha)^{\frac{1}{2}}(\lambda x+\mu),
$$

where $\mu$ is a constant of integration. It may readily be verified that if we define new constants $M, N, A$ and $B$ by the relations

$$
\begin{aligned}
& M=\left[\frac{(g \alpha \lambda)^{2}}{16 \alpha\left(\tilde{b}_{1}^{2}\right)^{4}}\right]^{\frac{1}{6}}, \\
& N=\frac{\delta(g \alpha)^{\frac{1}{2}}}{2 \tilde{b}_{1}^{2} M} \\
& A=\frac{5(g \alpha)^{\frac{1}{2}} \lambda}{8\left(\tilde{b}_{1}^{2}\right)^{2} M^{5}} \\
& B=\frac{5(g \alpha)^{\frac{1}{2}} \mu}{8\left(\tilde{b}_{1}^{2}\right)^{2} M^{5}}
\end{aligned}
$$

and also define a new parameter $\theta$ by $x^{*}=M^{2} \theta^{2}$ then (3.29) and (3.30) yield, in turn,

$$
\begin{gathered}
h_{0}=\theta^{2}[\theta+N]^{4}, \\
\theta^{3}\left[\theta^{2}+\frac{5}{2} N \theta+\frac{5}{3} N^{2}\right]=A x+B
\end{gathered}
$$


It should be noted that, regardless of the value taken by $N$, the curve given by (3.35) and (3.36) can, in general, only be fitted through two points $\left(x_{i}, h\left(x_{i}\right)\right]$ for $i=1,2$. For the case $N=0$ (corresponding to $\delta=0$ ) the parameter $\theta$ may be eliminated to give the explicit relation

$$
h_{0}=(A x+B)^{\frac{6}{3}} .
$$

Summarising, it has been shown that when the bottom has the curvilinear shape defined by (3.27), the governing equations may be reduced to those associated with long wave propagation due to ground motion in the constant depth situation (see Kajiura [1]). Further it has been established that when the bottom has the shape defined by (3.35) and (3.36), the governing equations may be transformed to those descriptive of long wave propagation due to ground motion in the uniform slope situation. Thus the analysis of Tuck and Hwang [2] becomes available.

\section{(b) NO GROUND MOTION}

In this case it follows from (3.24) that reduction to canonical form (i) may be achived when $a ;$ adopts one of the forms

$$
\begin{aligned}
& a_{1}^{1}=1 /\left(\alpha x^{*}+\gamma\right), \quad \beta=0, \\
& a_{1}^{1}=-\beta x^{*}+\delta, \quad \alpha=0, \\
& a_{1}^{1}=(\beta / \alpha)^{\frac{1}{2}} \cot \left\{(\beta / \alpha)^{\frac{1}{2}}\left(\alpha x^{*}+\zeta\right)\right\}, \quad \beta / \alpha>0, \\
& a_{1}^{1}=(-\beta / \alpha)^{\frac{1}{2}} \tanh \left\{(-\beta / \alpha)^{\frac{1}{2}}\left(\alpha x^{*}+\eta\right)\right\}, \quad \beta / \alpha<0,
\end{aligned}
$$

where $\alpha=\tilde{b} \frac{1}{2} \lambda^{-1}, \beta=-\tilde{b}_{1}^{2}$ and $\gamma, \delta, \zeta, \eta$ are arbitrary constants. The case (3.39) is, of course, the same as (3.26). The bottom profiles corresponding to (3.38) and (3.39) are, respectively,

$$
h_{0}=(A x+B)^{4}, \quad h_{0}=(A x+B)^{\frac{4}{3}},
$$

where, in each case, the constants $A$ and $B$ are suitably related to the constants occurring in (3.38) and (3.39). The forms for $h_{0}$ corresponding to (3.40) and (3.41) are

$$
\begin{aligned}
& h_{0}=\left(\beta^{2} / \alpha^{2} \lambda^{2} g\right) \cot ^{4}\left\{(\beta / \alpha)^{\frac{1}{2}}\left(\alpha x^{*}+\zeta\right)\right\}, \\
& h_{0}=\left(\beta^{2} / \alpha^{2} \lambda^{2} g\right) \tanh ^{4}\left\{(-\beta / \alpha)^{\frac{1}{2}}\left(\alpha x^{*}+\eta\right)\right\},
\end{aligned}
$$

where the $\left(x^{*}, x\right)$-relation is given by $(2.15)$.

Reduction to canonical form (ii) is only considered in the cases $\tilde{b}_{2}^{1}=0$ and $\tilde{b}_{1}^{2}=0$. The former case simply leads to $a_{1}^{1}$ in the form (3.28), while the case $\tilde{b}_{1}^{2}=0$ gives

$$
a_{1}^{1}=\left\{\left[2(g \alpha)^{\frac{1}{2}} \tilde{b}_{2}^{1} / 3 \lambda\right] x^{* \frac{3}{2}}+\mu\right\}^{-1} .
$$


where $\mu$ is an arbitrary constant of integration. It is noted that the solution of the Riccati equation (3.24) with $h_{2}^{\prime \prime}, h_{1}^{2 \prime}$ defined by (3.3) and $\bar{b}_{2}^{1} \neq 0, \bar{b}_{1}^{2} \neq 0$ may be obtained by transformation to an equation amenable to the Frobenius method. Equation (3.46) corresponds to a reduction to canonical form (ii) when

$$
h_{0}=\alpha x^{*}\left\{\lambda^{2}\left[\left(2(g \alpha)^{\frac{1}{2}} \tilde{b}_{2}^{1} / 3 \lambda\right) x^{* \frac{3}{2}}+\mu\right]^{4}\right\}^{-1},
$$

where the $\left(x^{*}, x\right)$-rélation is âgain given by (2.15). In particulâr, if $\mu=0$, this yields

$$
h_{0}=(A x+B)^{\frac{10}{3}},
$$

where $A$ and $B$ are arbitrary constants.

\section{Reduction for more general classes of $h_{0}(x)$.}

It is natural to enquire whether reduction to canonical forms (i), (ii) may be established for $h_{0}(x)$ other than those noted above. In this section, two methods are suggested for extending the scope of the method presented in this paper. The first approach is similar to one used by Clements and Rogers [4] in the context of wave propagation through inhomogeneous elastic materials, while the second, involving iteration of what is essentially Weinstein's [6] correspondence principle, has been discussed by Rogers [7] in connection with the propagation of large amplitude disturbances through certain non-linear elastic media.

If we return to the original system (2.7), (2.8) and eliminate $u$, it is seen that

$$
\frac{\partial^{2} \eta}{\partial t^{2}}-\frac{\partial}{\partial x}\left[g h_{0} \frac{\partial \eta}{\partial x}\right]=\frac{\partial^{2} \eta_{0}}{\partial t^{2}} .
$$

It is now required that (4.1) be generated as a consequence of the matrix equation

where

$$
\mathbf{\Omega}_{x}=\mathcal{M} \mathbf{\Omega}_{\mathrm{t}}+\mathcal{N} \mathbf{\Omega}+\boldsymbol{\theta}_{t}
$$

$$
\begin{aligned}
& \boldsymbol{\Omega}=\left(\begin{array}{l}
\eta \\
\varepsilon
\end{array}\right), \\
& \mathcal{M}=\left(\begin{array}{cc}
0 & m_{12} \\
m_{21} & 0
\end{array}\right), \\
& \mathcal{N}=\left(\begin{array}{cc}
n_{11} & 0 \\
0 & 0
\end{array}\right), \\
& \boldsymbol{\theta}=\left(\begin{array}{c}
0 \\
-m_{21} \eta_{0}
\end{array}\right)
\end{aligned}
$$

and where $m_{12}, m_{21}$ and $n_{11}$ are dependent on $x$ alone. The requirement that elimination of the 'intermediate' variable $\varepsilon(x, t)$ yield $(4.1)$ shows that 


$$
\begin{aligned}
m_{12} m_{21} & =1 /\left(g h_{0}\right), \\
h_{0} m_{12} & =C_{1} \exp \left\{-\int n_{11} d x\right\}, \\
n_{11} / m_{12} & =C_{2},
\end{aligned}
$$

where $C_{1}$ and $C_{2}$ are constants. If we set

$$
\Phi=C_{1} \exp \left\{-\int n_{11} d x\right\}
$$

(4.7)-(4.9) may be rewritten in the form

$$
\begin{aligned}
& m_{21}=\Phi / h_{0}, \\
& m_{21}=1 / g \Phi, \\
& \Phi^{-1}=\int\left(C_{2} / h_{0}\right) d x+C_{3}
\end{aligned}
$$

where $C_{3}$ is a further constant of integration. Thus, if $h_{0}(x)$ is specified, $\Phi$ may be determined from (4.13) and then (4.11), (4.12) and (4.9) yield $m_{12}, m_{21}$ and $n_{11}$. Alternatively, given a particular $\Phi,(4.13)$ yields $h_{0}$ and (4.11), (4.12) and (4.9) $m_{12}, m_{21}$ and $n_{11}$.

The transformations

$$
\begin{aligned}
\eta^{*} & =g^{-1} \Phi \eta \\
\varepsilon^{*} & =\varepsilon
\end{aligned}
$$

reduce $(4.2)$ to the system

$$
\boldsymbol{\Omega}_{x}^{*}=\mathcal{M}^{*} \boldsymbol{\Omega}_{i}^{*}+\boldsymbol{\theta}_{\boldsymbol{i}}
$$

where

$$
\begin{aligned}
& \mathbf{\Omega}^{*}\left(\begin{array}{l}
\eta^{*} \\
\varepsilon^{*}
\end{array}\right), \\
& \mathcal{M}^{*}=\left(\begin{array}{cc}
0 & \Phi^{2} / g h_{0} \\
1 / \Phi^{2} & 0
\end{array}\right),
\end{aligned}
$$

Introduction of the new variables $x^{*}, t^{*}$ defined by $(2.15),(2.16)$ reduces $(4.16)$ to

$$
\boldsymbol{\Omega}_{x^{*}}^{*}=\boldsymbol{H}^{*} \boldsymbol{\Omega}_{\mathrm{i} *}^{*}+\boldsymbol{\theta}_{\mathrm{t} *}^{*},
$$

where

$$
\begin{aligned}
\boldsymbol{H}^{*} & =\left(\begin{array}{cc}
0 & \Phi^{2} /\left[g h_{0}\right]^{\frac{1}{2}} \\
{\left[g h_{0}\right]^{\frac{1}{2}} / \Phi^{2}} & 0
\end{array}\right), \\
\boldsymbol{\theta}^{*} & =\left(\begin{array}{c}
0 \\
-\left[g h_{0}\right]^{\frac{1}{2}} m_{21} \eta_{0}
\end{array}\right) .
\end{aligned}
$$


Procedures analogous to those of the preceding section (the particular case $n_{11} \equiv 0$ ) may now be used to obtain reduction to either of the forms (i) or (ii) for more general expressions for $h_{0}(x)$. The expressions for $h_{0}(x)$ are given by

$$
g h_{0}=(a !)^{4} \Phi^{4} / \lambda^{2},
$$

where $a_{1}^{1}$ adopts the forms given in section 3. As an illustration, from (3.38) and (4.22), it is seen that reduction to the canonical form (i) is possible, in particuiar, when

$$
h_{0}=\left(g \lambda^{2}\right)^{-1}\left[\alpha x^{*}+\gamma\right]^{-4} \Phi^{4} .
$$

Now, (2.15) and (4.13) show that

$$
\Phi=C_{4}\left[\alpha x^{*}+\gamma\right]^{3}+C_{5},
$$

so that

$$
h_{0}=\frac{\left\{C_{4}\left[\alpha x^{*}+\gamma\right]^{3}+C_{s}\right\}^{4}}{g \lambda^{2}\left[\alpha x^{*}+\gamma\right]^{4}}
$$

where $C_{4}$ and $C_{5}$ are constants. Writing $\alpha x^{*}+\gamma=\theta$ and using (2.15), it is apparent that $h_{0}$ is given parametrically by

$$
\begin{gathered}
h_{0}=\frac{\left[C_{4} \theta^{3}+C_{5}\right]^{4}}{g \lambda^{2} \theta^{4}}, \\
\frac{1}{5} C_{4}^{2} \theta^{5}+C_{4} C_{5} \theta^{2}-C_{5} \theta^{-1}=\alpha \lambda x+\mu .
\end{gathered}
$$

The cases $C_{4}=0$ and $C_{5}=0$ yield, in turn,

$$
h_{0}=(A x+B)^{4}, \quad h_{0}=(A x+B)^{\frac{f}{3}},
$$

where, in each case, the relationship between $A, B$ and $C_{4}, C_{5}, \alpha, \lambda, \mu$ may readily be obtained.

As another method for obtaining reduction to canonical form, the transformations may be iterated. For illustrative purposes, attention is confined to seeking $h_{0}(x)$ for which reduction to canonical form (i) may be made in the absence of ground motion. Thus, reduction to the conventional wave equation is sought.

Consider the transformations

$$
\begin{aligned}
& \boldsymbol{\Omega}_{y}=\boldsymbol{A}_{1} \boldsymbol{\Omega}_{\mathbf{y}}^{\prime}+\boldsymbol{B}_{1} \boldsymbol{\Omega}^{\prime}, \\
& \boldsymbol{\Omega}_{\mathfrak{t}}=\boldsymbol{A}_{2} \boldsymbol{\Omega}_{\mathfrak{t}}^{\prime}+\boldsymbol{B}_{2} \boldsymbol{\Omega}^{\prime},
\end{aligned}
$$

where

$$
\boldsymbol{A}_{1}=\boldsymbol{A}_{2}=\left(\begin{array}{cc}
0 & y^{-p-1} \\
y^{p+1} & 0
\end{array}\right)
$$




$$
\begin{aligned}
& \boldsymbol{B}_{1}=\left(\begin{array}{cc}
0 & 0 \\
(p+1) y^{p} & 0
\end{array}\right), \\
& \boldsymbol{B}_{2}=\left(\begin{array}{cc}
(p+1) & 0 \\
0 & 0
\end{array}\right) .
\end{aligned}
$$

These transformations link the system

$$
\begin{gathered}
\boldsymbol{\Omega}_{y}^{\prime}=\boldsymbol{H}^{\prime} \boldsymbol{\Omega}_{t}^{\prime}, \\
\boldsymbol{H}^{\prime}=\left(\begin{array}{cc}
0 & y^{-p-2} \\
y^{p+2} & 0
\end{array}\right),
\end{gathered}
$$

with the associated system

$$
\begin{gathered}
\boldsymbol{\Omega}_{\boldsymbol{y}}=\boldsymbol{H} \boldsymbol{\Omega}_{\boldsymbol{t}}, \\
\boldsymbol{H}=\left(\begin{array}{cc}
\mathbf{0} & \boldsymbol{y}^{-p} \\
y^{p} & 0
\end{array}\right),
\end{gathered}
$$

where

$$
\mathbf{\Omega}^{\prime}=\left(\begin{array}{l}
\eta^{\prime} \\
u^{\prime}
\end{array}\right), \quad \mathbf{\Omega}=\left(\begin{array}{l}
\eta \\
u
\end{array}\right)
$$

Explicitly, the transformations yield

$$
\begin{aligned}
\eta_{7} & =y^{-p-1} u_{t}^{\prime}+(p+1) \eta^{\prime}, \quad p \neq-1 \\
\eta_{y} & =y^{-p-1} u_{t}^{\prime}, \\
u & =y^{p+1} \eta^{\prime} .
\end{aligned}
$$

The latter gives an analogue for the hyperbolic system (4.31), (4.32) of Weinstein's [6] correspondence principle for the Stokes-Beltrami equations.

It is readily shown by iteration of the above transformation that the general solution of the system (4.31), (4.32) for $p=2 N, N=0,1,2, \cdots$ is given by

$$
\begin{aligned}
\eta & =\sum_{r=0}^{N} \frac{(-1)^{r} 2^{r}(2 N-r) ! y^{-2 N+r} r}{r !(N-r) !(2 N-r)}\left[F^{(r+1)}(t+y)+(-1)^{r} G^{(r+1)}(t-y)\right], \\
& \equiv f(y, t), \\
u & =\sum_{r=0}^{N} \frac{(-1)^{r} 2^{r}(2 N-r) ! y^{r}}{r !(N-r) !}\left[F^{(r+1)}(t+y)-(-1)^{r} G^{(r+1)}(t-y)\right] \\
& \equiv g(y, t)\left(\frac{r}{2 N-r}=1 \quad \text { when } \quad r=N=0\right),
\end{aligned}
$$

where $F, G$ are arbitrary functions of $t+y$ and $t-y$ respectively, while $\Phi^{(r)}(\zeta)$ denotes the $r$ th derivative of $\Phi$ with respect to its indicated argument $\zeta$. It is further observed that the single transformation 


$$
\eta=u^{\dagger}, \quad u=\eta^{\dagger}
$$

takes the system (4.31) to the system

$$
\begin{gathered}
\boldsymbol{\Omega}_{y}^{\dagger}=\boldsymbol{H}^{\dagger} \boldsymbol{\Omega}_{\mathrm{t}}^{\dagger}, \\
\boldsymbol{H}^{\dagger}=\left(\begin{array}{cc}
0 & y^{p} \\
y^{-p} & 0
\end{array}\right), \\
\boldsymbol{\Omega}^{\dagger}=\left(\begin{array}{l}
\eta^{\dagger} \\
u^{+}
\end{array}\right) .
\end{gathered}
$$

so that the solution of the system (4.31) and (4.32) for $p=-2 N, N=$ $0,1,2, \cdots$ is given by

$$
\begin{gathered}
\eta=g(y, t), \\
u=f(y, t),
\end{gathered}
$$

where $f, g$ are as defined in (4.36), (4.37) above.

By comparing (2.17)-(2.20) and (4.31, (4.32) it is apparent that if

$$
h_{0}(x)=g^{-1}[(p+1) x]^{\frac{20}{p+1}},
$$

then, in the absence of ground motion, the system (2.7), (2.8) has the solution

$$
\begin{aligned}
\eta & =f(y, t), \\
u h_{0} & =-g(y, t), \quad \text { for } p=0,2,4, \cdots
\end{aligned}
$$

and

$$
\begin{aligned}
\eta & =g(y, t), \\
u h_{0} & =-f(y, t) \quad \text { for } p=0,-2,-4, \cdots
\end{aligned}
$$

where $f$ and $g$ are defined by (4.36) and (4.37) and

$$
y=[(p+1) x]^{\frac{1}{p+1}}
$$

\section{Solutions to the basic equations}

The results of the previous sections are now used to obtain specific solutions to the basic equations. In order to demostrate the technique it will be sufficient to consider bottom profiles which are special cases of (3.27) and (3.37).

Eliminating $u$ from (2.7) and (2.8) we obtain

$$
\frac{\partial^{2} \eta}{\partial t^{2}}-g \frac{\partial}{\partial x}\left[h_{0} \frac{\partial \eta}{\partial x}\right]=\frac{\partial \eta_{0}}{\partial t} .
$$


We consider the bottom profiles $h_{0}=x^{\frac{4}{3}}$, and $h_{0}=x^{\frac{6}{3}}$ separately.

(i)

$$
h_{0}(x)=x^{4} .
$$

In this case (3.27) is applicable with $A=1$ and $B=0$. Use of (3.4), (3.5) and (3.26) yields

$$
\begin{aligned}
& \eta_{x^{*}}^{\prime}=\left(\tilde{b}_{1}^{2} x^{*}+\delta\right) \eta_{x^{*}}^{*}+\tilde{b}_{1}^{2} \eta^{*}, \\
& \eta_{i^{*}}^{\prime}=\left(\tilde{b}_{1}^{2} x^{*}+\delta\right) \eta_{i}^{*} .
\end{aligned}
$$

Hence, integrating and using (2.10) and $(2,15)$, we obtain

$$
\eta^{\prime}=\tilde{b}_{i}^{2} x^{*} \eta, \quad x^{*}=3 g^{-\frac{1}{2}} x^{\frac{1}{3}},
$$

where the constant $\delta$ is necessarily zero because $x=0$ when $x^{*}=0$. The constant $\tilde{b}_{1}^{2}$ may be chosen arbitrarily so, for convenience, take $\tilde{b}_{1}^{2}=1$. Hence, the transformations

transform (5.1) to

$$
\begin{aligned}
& \eta^{\prime}=3 g^{-\frac{1}{2}} x^{\frac{1}{3}} \eta, \\
& x^{*}=3 g^{-\frac{1}{2}} x^{\frac{1}{3}}
\end{aligned}
$$

$$
\frac{\partial^{2} \eta^{\prime}}{\partial t^{2}}-\frac{\partial^{2} \eta^{\prime}}{\partial x^{* 2}}=x * \frac{\partial \eta_{0}}{\partial t}
$$

Putting. $u=\left(x^{*}+t\right) / 2, v=\left(x^{*}-t\right) / 2$ in (5.5) it follows that

$$
\frac{\partial^{2} \eta}{\partial u \partial v}=-x^{*} \bar{\eta}_{0}
$$

where $\bar{\eta}_{0}=\partial \eta_{0} / \partial t$. Integrating

$$
\eta^{\prime}=F(u)+G(v)-\iint(u+v) \bar{\eta}_{0} d u d v
$$

where $F$ and $G$ are arbitrary functions of their respective arguments. Using (5.3), (5.4) and (5.7) the general solution to (5.1) (with $h_{0}$ given by (5.2)) may be written in the form

$$
\eta=(1 / 3) g^{\frac{1}{2}} x^{-\frac{1}{3}}\left\{F(u)+G(v)-\iint(u+v) \bar{\eta}_{0} d u d v\right\}
$$

where

$$
\begin{gathered}
u=\frac{1}{2}\left(3 g^{-\frac{1}{2}} x^{\frac{1}{3}}+t\right), \\
v=\frac{1}{2}\left(3 g^{-\frac{1}{2}} x^{\frac{1}{3}}-t\right) . \\
h_{0}(x)=x^{\frac{6}{3} .}
\end{gathered}
$$

In this case (3.37) is applicable with $A=1, B=0$. Use of (3.4), (3.5) and (3.26) yields

$$
\begin{aligned}
& \eta_{x^{*}}^{\prime}=\left[2(g \alpha)^{-\frac{1}{2}} \tilde{b}_{1}^{2} x^{* \frac{1}{2}}+\delta\right] \eta_{x^{*}}^{*}+\left(g \alpha x^{*}\right)^{-\frac{1}{2}} \tilde{b}_{1}^{2} \eta^{*}, \\
& \left.\eta_{i^{*}}^{\prime}=[2 g \alpha)^{-\frac{1}{2}} b_{1}^{2} x^{* \frac{1}{2}}+\delta\right] \eta_{1}^{*} .
\end{aligned}
$$


Integrating and using (2.10) and (2.15) we obtain

$$
\eta^{\prime}=2(g \alpha)^{-\frac{1}{2}} \tilde{b}_{i}^{2} x^{* \frac{1}{2}} \eta, \quad x^{*}=(5 / 2) g^{-\frac{1}{2}} x^{\frac{2}{3}},
$$

where, as in case (i), $\delta$ is necessarily zero since $x=0$ when $x^{*}=0$. The constant $\tilde{b}_{1}^{2}$ may be chosen arbitrarily so, for convenience, put $\bar{b}_{1}^{2}=\frac{1}{2}(g \alpha)^{\frac{1}{2}}$. Thus the transformations

$$
\begin{aligned}
\eta^{\prime} & =x^{* \frac{1}{2}} \eta, \\
x^{*} & =(5 / 2) g^{-\frac{1}{2}} x^{\frac{2}{3}}
\end{aligned}
$$

transform (5.1) to

$$
\frac{\partial^{2} \eta^{\prime}}{\partial t^{2}}-\frac{1}{x^{* \frac{1}{2}}} \frac{\partial}{\partial x^{*}}\left[x^{* \frac{1}{2}} \frac{\partial \eta^{\prime}}{\partial x^{*}}\right]=x^{* \frac{1}{2}} \frac{\partial \eta_{0}}{\partial t},
$$

Introduction of the new independent variable

$$
\bar{x}=(2 / 3) x^{* \frac{3}{2}}=(2 / 3)\left[(5 / 2) g^{-\frac{1}{2}}\right]^{\frac{3}{2}} x^{\frac{3}{3}}
$$

transforms (5.15) to

$$
\frac{\partial^{2} \eta^{\prime}}{\partial t^{2}}-\frac{\partial}{\partial \bar{x}}\left[\bar{x} \frac{\partial \eta^{\prime}}{\partial \bar{x}}\right]=(3 \bar{x} / 2)^{\frac{1}{3}} \frac{\partial \eta_{0}}{\partial t} .
$$

The solution this equation may be expressed in terms of integrals involving Bessel functions (see Tuck and Hwang [2]).

\section{References}

[1] K. Kajiura, 'The leading wave of a tsunami', Bull. Earthquake Res. Inst. 41 (1963), 535.

[2] E. O. Tuck and L.-S. Hwang, 'Long wave generation on a sloping beach', J. Fluid Mech. 51 (1972), 449.

[3] D. L. Clements and C. Rogers, 'Wave propagation in inhomogeneous elastic media', Int. J. Solids Structures 10 (1974), 661.

[4] D. L. Clements and C. Rogers, 'Wave propagation in inhomogeneous elastic media with $(N+1)$-dimensional spherical symmetry', Canadian J. Physics 52 (1974), 1246.

[5] C. Rogers and D. L. Clements, 'A note on the reduction of the hodograph equations for one-dimensional elastic-plastic wave propagation', Quart. Appl. Math. 32 (1975), 469.

[6] A. Weinstein, 'Generalized axially symmetric potential theory', Bull. Amer. Math. Soc. 59 (1953), 20.

[7] C. Rogers, 'Iterated Baecklund-type transformations and the propagation of disturbances in non-linear elastic materials', J. Math. Anal. Applics. (to appear).

Department of Applied Mathematics,

University of Adelaide, Australia

and

S.L.I.S. University of Western Ontario,

London, Canada. 